\title{
Association Between Vascular Overload Index and New-Onset Ischemic Stroke in Elderly Population with Hypertension
}

\author{
Yuling Yu $\mathbb{D}^{1, *}$ \\ Yuqing Huang ${ }^{1, *}$ \\ Lin Liu $^{2}$ \\ Xiao-Cong Liu (iD) \\ Qiuhong $\operatorname{Tan}^{3}$ \\ Chaolei Chen (ID) \\ Jiayi Huang ${ }^{3}$ \\ Yingqing Feng' \\ Songtao Tang ${ }^{4}$
}

'Department of Cardiology, Guangdong Cardiovascular Institute, Guangdong Provincial People's Hospital, Guangdong Academy of Medical Sciences,

Guangzhou, 510080, People's Republic of China; ${ }^{2}$ The Second School of Clinical Medicine, Southern Medical University, Guangzhou, People's Republic of China; ${ }^{3}$ Guangdong Provincial People's Hospital, School of Medicine, South China University of Technology, Guangzhou, People's Republic of China; ${ }^{4}$ Department of Cardiology, Community Health Center of Liaobu Town, Dongguan, 523400 , Guangdong, People's Republic of China

*These authors contributed equally to this work

Correspondence: Songtao Tang Department of Cardiology, Community Health Center of Liaobu Town, Dongguan, 523400, Guangdong, People's Republic of China

Email3038384760@qq.com

Yingqing Feng

Department of Cardiology, Guangdong

Cardiovascular Institute, Guangdong

Provincial People's Hospital, Guangdong

Academy of Medical Sciences, 106 2nd

Zhongshan Road, Yuexiu District,

Guangzhou, 5I 0080, People's Republic of

China

Email fengyingqing@gdph.org.cn
Background: Vascular overload index (VOI) is a marker of arterial stiffness and arteriolar resistance, which predicts the increasing risks of cardiovascular and cerebrovascular disease. This study aimed to evaluate the association between VOI and new-onset ischemic stroke in an elderly population with hypertension.

Methods: This retrospective cohort study included 3315 hypertensive participants aged 60 years or more. Ischemic stroke was diagnosed according to cranial computed tomography, magnetic resonance imaging of the brain or cerebrovascular angiography. The calculation of VOI was based on systolic and diastolic blood pressure. VOI was divided by quartiles $(<7.88$ $\mathrm{mmHg}, 7.88-16.10 \mathrm{mmHg}, 16.10-27.14 \mathrm{mmHg}, \geq 27.14 \mathrm{mmHg}$ ) and evaluated the association with new-onset ischemic stroke by multivariable Cox regression models.

Results: A total of 3315 participants (55.5\% female) aged $71.4 \pm 7.20$ years were included in the analysis. The median follow-up period was 5.5 years, and 206 participants reached the endpoint, new-onset ischemic stroke. With per standard deviation increment in VOI, the risks of new-onset ischemic stroke increased in non-adjusted model (Hazard ratio [HR], 1.11; 95\% confidence interval [CI]: 1.03-1.22; $p=0.001)$, adjusted model (HR, 1.11; 95\% CI: 1.04$1.22 ; p=0.003$ ) and fully-adjusted model (HR, 1.15; 95\% CI: 1.08-1.26; $p<0.001$ ), respectively. In multivariate fully adjusted model, the risks of ischemic stroke increased in higher quartiles in comparison to the first quartiles ( $p$ for trend $<0.001$ ).

Conclusion: In an elderly hypertensive population, VOI is significantly associated with the incidence of new-onset ischemic stroke. Elevated VOI is the cardiovascular risk factor and increases the probability of new-onset ischemic stroke.

Keywords: vascular overload index, ischemic stroke, hypertension, elderly population, blood vessel

\section{Introduction}

Blood pressure (BP) is a non-invasive parameter reflecting a primary rise in pressure caused by left ventricular ejection and a secondary rise in pressure of blood in the circulation system against the walls of distal blood vessels. ${ }^{1}$ Among elderly populations, increased BP is one of the risk factors inducing hypertensionmediated organ damage including stroke, ${ }^{2,3}$ with the pathophysiological mechanism of arterial stiffness and decreased elasticity of blood vessels. ${ }^{1,3}$ Arterial stiffness is hypothesized to contribute to cerebral microvascular damage, cognitive impairment and dementia by reducing mean cerebral blood flow and increasing pulsatile stress in the brain. ${ }^{4}$ Current guidelines ${ }^{5,6}$ and various studies ${ }^{7,8}$ agree that higher systolic 
$\mathrm{BP}$ is associated with cardiovascular and cerebrovascular risks after adjustment for, or within stratums of diastolic BP, while non-significant association was shown between diastolic BP and cardiovascular risk with the adjustment or stratification of systolic BP, which indicates systolic BP might be more capable for the prognosis of adverse outcomes. Moreover, the Framingham Heart Study demonstrated that systolic rather than diastolic BP is a optimal risk marker for stroke among the population aged 45 years or more. ${ }^{9-11}$ However, diastolic BP is an important indicator of elasticity of blood vessels. ${ }^{12}$ Vascular overload index (VOI), calculated by both components of BP measurement and mainly accounted for by systolic BP, is a possible comprehensive parameter related to the cardiovascular and cerebrovascular risk in consideration of increased arteriolar resistance, increased large-artery stiffness, and early or premature reflection of arterial pulse waves. ${ }^{13}$ Previous studies provided limited evidence to demonstrate the association between evaluated VOI and cardiovascular risks, ${ }^{14,15}$ and the relationship of VOI with ischemic stroke remains uncertain in Chinese population. Therefore, we administrated a longitudinal observational cohort study to evaluate the association of VOI and ischemic stroke in an elderly hypertensive population based on office BP measurement.

\section{Methods}

\section{Study Design and Population}

This was a retrospective cohort study conducted in Liaobu community in Guangdong, China. This study was approved by the institutional medical ethical committee of Guangdong Provincial People's Hospital, Guangzhou, China (No. 2012143H). All procedures performed in this study were in accordance with the ethical standards of the 1964 Helsinki Declaration and its later amendments or comparable ethical standards. Informed consent was obtained from all individual participants included in this study.

In this study, the inclusion criteria were as follows: (i) participants with essential hypertension; (ii) aged 60 years or more; (iii) participated in the baseline examination in 2010 and follow-up visits; (iv) gave informed consent before baseline. Besides, the exclusion criteria were: (i) participants with secondary hypertension; and (ii) history of ischemic stroke. A total of 3500 elderly hypertensive patients aged 60 years or more were recruited from January 1, 2010 to December 31, 2011. Baseline examinations and annual follow-up visits were administrated to obtain clinical and biochemical information. The duration of follow up started at the first visit and ended on December 31, 2016. Among all the participants, 185 were excluded due to previous history of stroke $(\mathrm{n}=$ 132), missing blood pressure records $(n=37)$ or other physical examinations $(\mathrm{n}=16)$. Finally, 3315 participants were included in statistical analysis (Figure 1).

\section{Clinical and Biochemical Measurements}

VOI is defined as a parameter calculated by both systolic blood pressure (SBP) and diastolic blood pressure (DBP). In accordance with the vascular overload concept, the formula of VOI calculation is as follows. ${ }^{13}$

$$
\mathrm{VOI}(\mathrm{mmHg})=1.33 \times \mathrm{SBP}-0.33 \times \mathrm{DBP}-133.3
$$

Blood pressure measurements were conducted according to the 2010 Chinese guidelines for management of hypertension. ${ }^{5}$ At the study sites, blood pressure measurements were taken by trained nurses or physicians. Participants were required to avoid exercise, smoking, and caffeine for at least 30 minutes and have a rest for longer than 5 minutes before measurement. Blood pressure was measured between 8 A.M. and 10 A.M. during the visit. The measured arm was positioned at the level of the heart and circled with cuffs of an appropriate size. Blood pressure was measured by an automated device (OMRONHBP1100u; Omron Corporation, Tokyo, Japan). The arm with higher brachial blood pressure was applied to all subsequent measurements and records. Hypertension was defined as an elevated blood pressure of SBP $\geq$ $140 \mathrm{~mm} \mathrm{Hg}$ and/or DBP $\geq 90 \mathrm{~mm} \mathrm{Hg}$, or receiving antihypertensive medication during the past two weeks. ${ }^{5}$

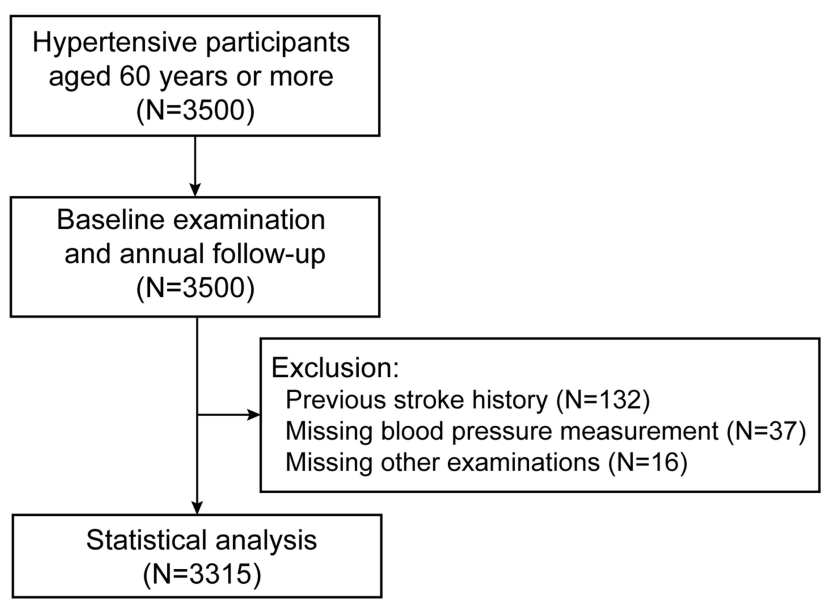

Figure I Flow chart of the study process, inclusion and exclusion criteria. 
Venous blood samples were obtained in the morning after 8-12 hours of fasting. Blood samples were detected immediately after collection. Serum total cholesterol, low density lipid cholesterol (LDL-C), high density lipid cholesterol (HDL-C), triglyceride and fasting blood glucose were detected by calibrated, multi-functional biochemical devices. Demographic and medical information, including age, sex, smoking status, alcohol consumption and medical history were obtained from self-reports or medical records. Diabetes mellitus was a self-reported diagnosis, with a fasting blood glucose of $7.0 \mathrm{mmol} / \mathrm{L}$ or higher, or the application of antidiabetic medication. Coronary artery disease was based on cardiovascular ischemia evidence including clinical symptoms, electrocardiogram and coronary artery angiography. Antihypertensive medications were classified as angiotensin converting enzyme inhibitor (ACEI), angiotensin receptor blocker (ARB) and calcium channel blocker (CCB). Antiplatelet drugs and statins were recorded. Body mass index (BMI) was calculated as the ratio of body weight in kilograms to the square of body height in meters $\left(\mathrm{kg} / \mathrm{m}^{2}\right)$. Estimated glomerular filtration rate (eGFR) was calculated by the simplified Modification of Diet in Renal Disease equation. ${ }^{16}$

\section{Clinical Outcome}

The primary endpoint of this study was defined as new-onset ischemic stroke, including cerebral infarction and transient ischemic attack. Clinical outcomes were ascertained from the local medical administrative bureau, which included the medical records of hospitalization and physical examinations. In the medical record, ischemic stroke was diagnosed according to the imaging evidence, including cranial computed tomography (CT) or contract vascular CT scan, magnetic resonance imaging of the brain, or cerebrovascular angiography, showing the evidence of obstruction or stenosis on the supplying blood vessel to the brain.

\section{Statistical Analysis}

The collected data were double entered to EpiData software 3.1 (EpiData Associations, Odense, Denmark). Statistical analysis was performed by $\mathrm{R}$ version 3.5.2 for windows (https://www.r-project.org/). Continuous variables were expressed as mean and standard deviation (SD), and categorical variables were presented as number and percentage. Departure from normality was evaluated by the Kolmogorov-Smirnov test. Skewness and kurtosis were computed as the third and fourth moments about the mean divided by the cube of the standard deviation. The differences between groups were evaluated by Student's $t$-test and ANOVA statistics for continuous variables or Chi-square test for categorical variables.

In exploratory analysis, VOI was expressed as a continuous variable and a categorical variable by quartiles. Hazard ratio (HR) and $95 \%$ confidence interval (95\% CI) were derived from Cox regression model. Unadjusted, adjusted and fully adjusted models were constructed to estimate the association between VOI (independent variable) and new-onset ischemic stroke (dependent variable). Unadjusted model adjusted for no covariates. Adjusted model included sex, age and BMI at baseline as covariates. Fully adjusted model additionally accounted for smoking status, alcohol consumption, SBP, total cholesterol, triglyceride, LDL-C, HDL-C, glucose, eGFR, diabetes mellitus, coronary artery disease, antihypertensive medication including ACEI, ARB and CCB. The covariates were selected according to the previously published literatures, which were associated to VOI (independent variable) and ischemic stroke (dependent variable). ${ }^{3,17-19}$ Sensitivity analysis was applied in subgroups by fully adjusted multivariate Cox regression model. Bonferroni correction was performed for multiple testing. ${ }^{20}$ The curves of HR and probability of ischemic stroke were generated with full adjustment. The threshold of statistical significance was defined as $p<0.05$.

\section{Results}

\section{Characteristics of Participants}

Baseline characteristics of all participants and in subgroups by quartiles in VOI are shown in Table 1. Of 3315 participants, 1475 (44.5\%) were male and 1840 (55.5\%) were female. At baseline, age averaged $71.4 \pm 7.20$ years, body mass index $23.9 \pm 3.80 \mathrm{~kg} / \mathrm{m}^{2}$, total cholesterol $11.3 \pm 2.53$ $\mathrm{mmol} / \mathrm{L}$, LDL-C $5.75 \pm 1.63 \mathrm{mmol} / \mathrm{L}$ and eGFR 98.7 $\pm 35.5 \mathrm{~mL} / \mathrm{min} / 1.73 \mathrm{~m}^{2}$. Among whom, 933 (28.1\%) participants are current smokers and 338 (10.2\%) consumed alcohol. $505(15.2 \%)$ were diagnosed with diabetes mellitus and $34(1.03 \%)$ had coronary artery disease. All continuous variables included in the analysis complied with normal distribution. Across the quartiles of the distribution of VOI, smoking status $(p=0.003)$, alcohol consumption $(p<0.001)$, age $(p<0.001)$, BMI $(p=0.018)$, SBP $(p<$ $0.001)$, DBP $(p<0.001)$, VOI $(p<0.001)$, triglyceride $(p=$ $0.021)$, ACEI $(p<0.001)$, ARB $(p<0.001), \operatorname{CCB}(p<$ $0.001)$ and history of coronary artery disease $(p=0.015)$ were significantly different among groups. 
Table I Baseline Characteristics of Participants Overall and in VOI by Quartiles

\begin{tabular}{|c|c|c|c|c|c|c|}
\hline & \multirow[t]{2}{*}{ Overall } & \multicolumn{4}{|c|}{ VOI by Quartiles (mm Hg) } & \multirow[t]{2}{*}{ p-value } \\
\hline & & $<7.88$ & $7.88-16.10$ & $16.10-27.14$ & $\geq 27.14$ & \\
\hline Number & 3315 & 806 & 851 & 825 & 833 & \\
\hline $\operatorname{Sex}(\%)$ & & & & & & 0.15 \\
\hline Male & $1475(44.5)$ & $371(46.0)$ & $391(45.9)$ & $370(44.8)$ & $343(4 I .2)$ & \\
\hline Female & $1840(55.5)$ & $435(54.0)$ & $460(54.1)$ & $455(55.2)$ & $490(58.8)$ & \\
\hline Smoking status (\%) & $933(28.1)$ & $258(32.0)$ & $252(29.6)$ & $222(26.9)$ & $201(24.1)$ & 0.003 \\
\hline Alcohol consumption (\%) & $338(10.2)$ & III (I3.8) & $86(10.1)$ & $81(9.82)$ & $60(7.20)$ & $<0.001$ \\
\hline Age (years) & $71.4(7.20)$ & $70.8(6.64)$ & $70.7(6.78)$ & $71.9(7.22)$ & $72.2(7.98)$ & $<0.001$ \\
\hline Body mass index $\left(\mathrm{kg} / \mathrm{m}^{2}\right)$ & $23.9(3.80)$ & $23.7(3.92)$ & $23.9(3.78)$ & $23.8(3.75)$ & $24.3(3.75)$ & 0.018 \\
\hline $\mathrm{SBP}(\mathrm{mm} \mathrm{Hg})$ & $131(17.2)$ & II $19.5(3.26)$ & $123(9.12)$ & $130(14.0)$ & $15 \mid(\mid 7.6)$ & $<0.001$ \\
\hline $\mathrm{DBP}(\mathrm{mm} \mathrm{Hg})$ & $78.3(9.55)$ & $75.0(6.93)$ & $76.9(6.89)$ & $78.3(9.48)$ & $83.2(11.9)$ & $<0.001$ \\
\hline VOI $(\mathrm{mm} \mathrm{Hg})$ & $20.0(16.6)$ & $3.00(2.32)$ & $11.9(2.22)$ & $21.8(3.04)$ & $43.1(14.0)$ & $<0.001$ \\
\hline Total cholesterol $(\mathrm{mmol} / \mathrm{L})$ & II.3 (2.53) & II.3 (2.52) & II.4 (2.55) & II.3 (2.64) & II.I (2.40) & 0.052 \\
\hline Triglyceride $(\mathrm{mmol} / \mathrm{L})$ & $8.33(6.44)$ & $8.23(6.43)$ & $8.00(6.15)$ & $8.17(6.22)$ & $8.92(6.91)$ & 0.021 \\
\hline LDL-C (mmol/L) & $5.75(1.63)$ & $5.65(1.58)$ & $5.82(1.69)$ & $5.73(1.59)$ & $5.80(1.67)$ & 0.14 \\
\hline HDL-C (mmol/L) & $2.82(0.78)$ & $2.82(0.76)$ & $2.81(0.69)$ & $2.83(0.89)$ & $2.81(0.76)$ & 0.95 \\
\hline Glucose $(\mathrm{mmol} / \mathrm{L})$ & $5.16(1.49)$ & $5.16(1.58)$ & $5.17(1.56)$ & $5.06(1.37)$ & $5.27(1.44)$ & 0.055 \\
\hline eGFR $\left(\mathrm{mL} / \mathrm{min} / 1.73 \mathrm{~m}^{2}\right)$ & $98.7(35.5)$ & I0I (38.3) & $97.4(32.5)$ & $96.7(36.6)$ & $95.2(32.4)$ & 0.17 \\
\hline ACEI (\%) & $212(6.40)$ & $4 \mid(5.09)$ & $39(4.58)$ & $55(6.67)$ & $77(9.24)$ & $<0.001$ \\
\hline ARB (\%) & $646(19.5)$ & $123(15.3)$ & $146(17.2)$ & $152(18.4)$ & $225(27.0)$ & $<0.001$ \\
\hline CCB (\%) & $656(19.8)$ & $123(15.3)$ & $135(15.9)$ & $152(18.4)$ & $246(29.5)$ & $<0.001$ \\
\hline Antiplatelet drugs (\%) & $197(5.94)$ & $48(5.96)$ & $64(7.52)$ & $40(4.85)$ & $45(5.40)$ & 0.11 \\
\hline Statin (\%) & $286(8.63)$ & $68(8.44)$ & $89(10.5)$ & $70(8.48)$ & $59(7.08)$ & 0.10 \\
\hline Diabetes mellitus (\%) & $505(15.2)$ & $136(16.9)$ & I4I (I6.6) & $115(13.9)$ & $113(13.6)$ & 0.13 \\
\hline Coronary artery disease (\%) & $34(1.03)$ & $12(1.49)$ & $4(0.47)$ & $14(1.70)$ & $4(0.48)$ & 0.015 \\
\hline
\end{tabular}

Notes: Data are presented as mean (standard deviation) for continuous variables and number (percentage) for categorical variables.

Abbreviations: SBP, systolic blood pressure; DBP, diastolic blood pressure; VOI, vascular overload index; LDL-C, low density lipid cholesterol; HDL-C, high density lipid cholesterol; eGFR, estimated glomerular filtration rate; ACEI, angiotensin converting enzyme inhibitor; ARB, angiotensin receptor blocker; CCB, calcium channel blocker.

With regard to the blood pressure, the averaged SBP/DBP were $131 \pm 17.2 / 78.3 \pm 9.55 \mathrm{mmHg}$ among all participants. Correspondingly calculated by SBP and DBP, mean VOI was $20.0 \pm 16.6 \mathrm{mmHg}$. Among the VOI quartiles $(<7.88$ $\mathrm{mmHg} ; 7.88-16.10 \mathrm{mmHg} ; 16.10-27.14 \mathrm{mmHg} ; \geq 27.14$ $\mathrm{mmHg}$ ), mean VOI were $3.00 \pm 2.32 \mathrm{mmHg}, 11.9 \pm 2.22$ $\mathrm{mmHg}, 21.8 \pm 3.04 \mathrm{mmHg}$ and $43.1 \pm 14.0 \mathrm{mmHg}$, respectively (Table 1).

\section{Clinical Outcome}

In a median follow-up period of 5.5 years (range interval, 5.0-7.0 years), a total of $206(6.21 \%)$ participants reached the endpoint, new-onset ischemic stroke. In the subgroups of VOI in quartiles, the mobility of newonset ischemic stroke was $4.96 \%, 5.64 \%, 6.06 \%$, and $8.16 \%$, respectively (Table 2). Across the quartiles of the distribution of VOI, the incidence of the endpoint, new-onset ischemic stroke increased significantly (Table 2, $p$ for trend $<0.001$ ).

\section{Association Between VOI and Ischemic Stroke}

In Cox regression models, association between VOI and new-onset ischemic stroke were both significant when VOI was expressed as continuous and categorical variables (Table 2). For all participants, as VOI increased per $\mathrm{SD}$ as continuous variable, it was significantly associated with new-onset ischemic stroke in non-adjusted (HR, 1.11; 95\% CI: $1.03-1.22 ; p=0.001$ ), adjusted (HR, 1.11; 95\% CI, 1.04-1.22; $p=0.003$ ), and fully adjusted models (HR, 1.15; 95\% CI: $1.08-1.26 ; p<0.001)$. The curves of HR and probability of new-onset ischemic stroke in VOI are shown with full adjustment of confounders in Figures 2 and 3 , respectively.

In addition, when VOI was applied as categorical variables by quartiles, the participants with higher levels of VOI positively associated with the incidence of ischemic stroke compared with the lowest quartile (all $p$ for trend $\leq 0.002$ ). Applied with fully adjusted model, the 
Table 2 Association Between VOI and Ischemic Stroke in Multivariate Cox Regression Model

\begin{tabular}{|c|c|c|c|c|c|c|c|c|c|c|}
\hline & \multirow[t]{2}{*}{$\mathbf{N}(\%)^{c}$} & \multicolumn{3}{|c|}{ Non-Adjusted $^{d}$} & \multicolumn{3}{|c|}{ Adjusted $^{d}$} & \multicolumn{3}{|c|}{ Fully Adjusted $^{d}$} \\
\hline & & HR & $95 \% \mathrm{Cl}$ & $p$-value & HR & $95 \% \mathrm{Cl}$ & $p$-value & HR & $95 \% \mathrm{Cl}$ & $p$-value \\
\hline $\mathrm{VOI}^{\mathrm{a}}$ & $206(6.21)$ & I.II & $(1.03-1.22)$ & 0.001 & 1.11 & $(1.04-1.22)$ & 0.003 & 1.15 & $(1.08-1.26)$ & $<0.001$ \\
\hline \multicolumn{11}{|l|}{ VOI by quartiles ${ }^{b}$} \\
\hline$<7.88 \mathrm{mmHg}$ & $40(4.96)$ & 1.0 & & & 1.0 & & & 1.0 & & \\
\hline $7.88-16.10 \mathrm{mmHg}$ & $48(5.64)$ & 1.12 & $(0.74-1.73)$ & 0.56 & I.II & $(0.74-I .72)$ & 0.58 & I.II & $(0.8 I-1.74)$ & 0.65 \\
\hline $16.10-27.14 \mathrm{mmHg}$ & $50(6.06)$ & 1.14 & (I.07-1.72) & 0.59 & 1.13 & $(1.08-1.80)$ & 0.63 & 1.34 & $(1.11-1.90)$ & 0.003 \\
\hline$\geq 27.14 \mathrm{mmHg}$ & $68(8.16)$ & 1.67 & $(1.15-2.13)$ & 0.028 & 1.71 & $(1.17-2.16)$ & 0.044 & 1.75 & $(1.18-2.17)$ & $<0.001$ \\
\hline$P$ for trend & $<0.001$ & \multicolumn{3}{|l|}{0.002} & \multicolumn{3}{|c|}{$<0.001$} & \multicolumn{3}{|c|}{$<0.001$} \\
\hline
\end{tabular}

Notes: ${ }^{a} \mathrm{VOI}$ was defined as continuous variable. The corresponding HRs represent the hazard ratio as per standard deviation of VOI increasing. ${ }^{\mathrm{b}} \mathrm{VOI}$ was defined as categorical variable by quartiles. The lowest quartile was reference group. The corresponding HRs represent the hazard ratio in different categories compared with reference group. ${ }^{\mathrm{C}} \mathrm{N}$ (\%) represents the number (percentage) of participants with new-onset ischemic stroke during follow up in total and in VOI categories. ${ }^{\mathrm{d}} \mathrm{HR}$ and $95 \% \mathrm{Cl}$ were derived from multivariate Cox regression models. Adjusted model accounted for age, sex and body mass index. Fully adjusted model additionally accounted for smoking status, alcohol consumption, systolic blood pressure, total cholesterol, triglyceride, low density lipid cholesterol, high density lipid cholesterol, glucose, estimated glomerular filtration rate, diabetes mellitus, coronary artery disease, antihypertensive medication including angiotensin converting enzyme inhibitor, angiotensin receptor blocker and calcium channel blocker.

Abbreviations: VOI, vascular overload index; HR, hazard ratio; $95 \% \mathrm{Cl}, 95 \%$ confidence interval.

relationship between VOI and ischemic stroke was ambiguous for the participants with VOI in second quartiles $(p=$ 0.65 ), whereas significant positive associations were shown in the third (HR, 1.34; 95\% CI: 1.11-1.10) and the highest quartile (HR, 1.75; 95\% CI: 1.18-2.17). In the highest quartile of VOI $\geq 27.14 \mathrm{mmHg}$, the associations were significant in non-, adjusted and fully adjusted models (all $p \leq 0.044$ ) (Table 2). The main result in fully adjusted Cox regression models was valid in multiple testing as well.

\section{Stratification Analysis in Subgroups}

The associations between VOI and ischemic stroke incidence were stratified by sex, smoking status, alcohol consumption, diabetes mellitus, SBP $<140$ and $\geq 140 \mathrm{mmHg}$, BMI $<25$ and $\geq 25 \mathrm{~kg} / \mathrm{m}^{2}$, hypertensive medication of ACEI, ARB and CCB (Table 3). Estimated by fully adjusted multivariate Cox regression models, the positive association between VOI and new-onset ischemic stroke were significant within above stratifications (all $p \leq 0.015$ ). The associations were not significantly different between subgroups (all $p$ for interaction $\geq 0.15$ ).

\section{Discussion}

\section{Interpretation of Current Study}

In our longitudinal study, among the elderly population aged 60 years or more, the elevated VOI at baseline was significantly associated with the higher HR and possibility of ischemic stroke in a median follow-up period of 5.5 years. Elevated VOI is the risk factor and prognostic marker of ischemic stroke, which represents the effective systolic blood pressure increment. ${ }^{13}$ As the current study was conducted in a hypertensive elderly population, various hypertensive medications and different physical status might cause uneven effect on blood pressure control. To exclude the interaction effects derived from the confounders, stratification analyses were applied in the subgroups of SBP $<140$ and $\geq 140 \mathrm{mmHg}$, BMI $<25$ and $\geq 25 \mathrm{~kg} / \mathrm{m}^{2}$, antihypertensive medication application and habitual smoking and alcohol consumption (Table 3), which proved to be consistent between stratums.

\section{VOI and Cardiovascular Risks}

Hypertension increases the prevalence of target organ damage in the brain, of which transient ischemic attack and stroke are the most dramatic acute clinical manifestations, and white matter hyperintensities and silent infarcts are pathophysiological process in asymptomatic phrase. ${ }^{3}$ In the current study, VOI represents the increase in arteriolar resistance and the increase in large-artery stiffness with resulting early-wave reflection that occur with aging and hypertension. ${ }^{13}$ In terms of the calculation formula, the value of VOI is mainly attributed to systolic BP and diastolic BP is the secondary component. The Systolic Hypertension in the Elderly Program (SHEP) enrolled 4736 participants aged 60 years or more with isolated systolic hypertension (systolic BP ranged 160-219 $\mathrm{mmHg}$; diastolic $\mathrm{BP}<90 \mathrm{mmHg}$ ) and randomized the participants to active treatment $(\mathrm{n}=2365)$ and placebo $(n=2371)$ groups. $^{21,22}$ The 5-year incidence of total stroke 


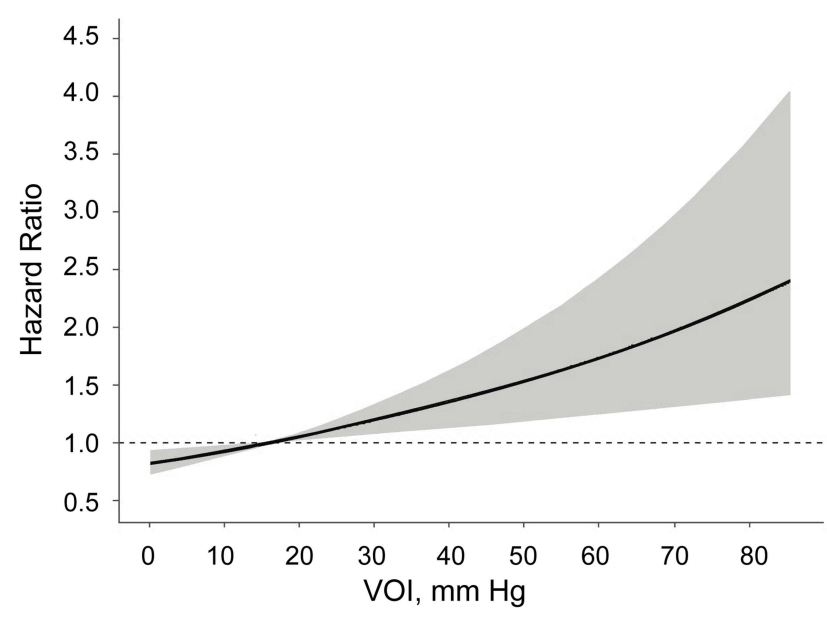

Figure 2 Hazard ratio and 95\% confidence interval of new-onset ischemic stroke in vascular overload index. Hazard ratio and $95 \%$ confidence interval were derived from fully adjusted Cox regression model, accounted for sex, age at baseline, BMI, smoking status, alcohol consumption, SBP, total cholesterol, triglyceride, LDL-C, HDL-C, glucose, eGFR, diabetes mellitus, coronary artery disease, antihypertensive medication including ACEI, ARB and CCB.

Abbreviation: $\mathrm{VOI}$, vascular overload index.

was 5.2 versus 8.2 per 100 participants in active treatment versus placebo (systolic BP: 143 vs $155 \mathrm{mmHg}$ ) with a relative risk by proportional hazards regression analysis of $0.64(p=0.0003) .{ }^{21}$ Similar to SHEP, the Systolic Blood Pressure Intervention Trial (SPRINT) was conducted in the hypertensive population aged 50 years or more, and observed a lower annual stroke incidence rate in intensive group $(0.41 \%)$ compared with standard treatment group $(0.47 \%)$ with a hazard ratio of 0.89 (95\% CI: $0.63-$ $1.25),{ }^{23}$ in line with the subgroup analyses results in the participants aged 75 years or more. ${ }^{24}$ These studies demonstrated that systolic BP is a more effective predictor of stroke and better prognosis can be achieved by effective control on systolic BP. Besides systolic BP, progressive elasticity on vessels wall, arterial stiffness and arteriolar resistance is associated with mean arterial pressure and diastolic $\mathrm{BP},{ }^{25-27}$ which indicates that vascular overload rather than BP parameters might be a better physiological measure to assess hypertensive cardiovascular and cerebrovascular risks. ${ }^{13}$

In a cross-sectional study that recruited 646 patients (386 male and 260 female) aged $62.2 \pm 12.3$ years with essential hypertension, the investigators measured the intima media thickness of carotid artery (CA-IMT) and echocardiogram to evaluate the association with VOI. ${ }^{14}$ Cases were categorized by the cut-off of IMT $<1.0 \mathrm{~mm}$ $(\mathrm{n}=376)$ and IMT $\geq 1.0 \mathrm{~mm}(\mathrm{n}=270)$, and 98 normotensive subjects were included in control group. ${ }^{14}$

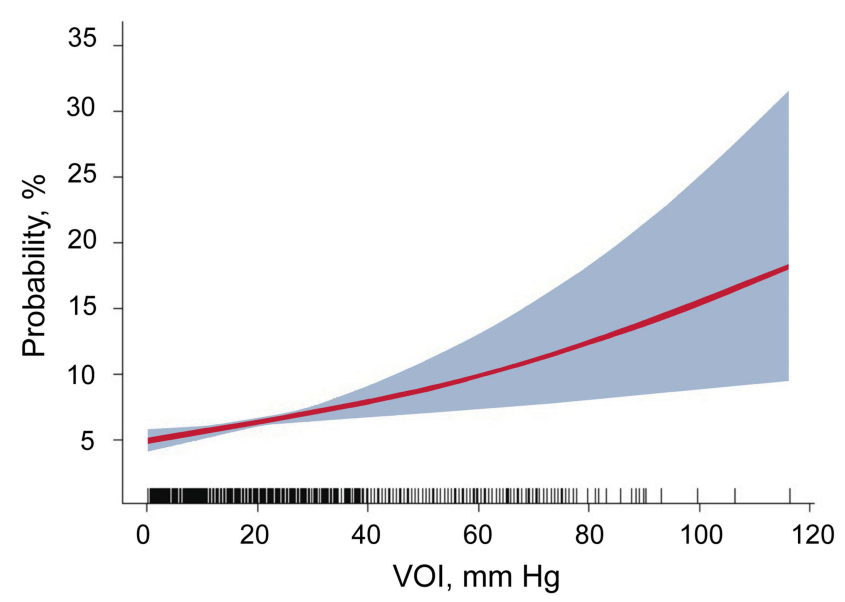

Figure 3 Probability and $95 \%$ confidence interval of new-onset ischemic stroke in vascular overload index.

Abbreviation: VOI, vascular overload index.

Compared with the lower IMT stratum, the participants with IMT $\geq 1.0 \mathrm{~mm}$ had an elevated VOI, left ventricular mass index and left-ventricular-mass-to-height ratio. Similar results were shown between hypertensive and control group. ${ }^{14}$ VOI was significantly associated with CA-IMT in multiple regression analysis after adjusted confounders. ${ }^{14}$ In order to explore the risk of increased VOI in different age groups, Wen and the co-author enrolled 102 middle-aged ( $<60$ years) and 104 elderly ( $\geq 60$ years) hypertensive participants matched for gender, body height, body weight, body mass index and body surface area, and measured blood pressure and echocardiogram. ${ }^{28}$ As compared with the middle-aged group, the elderly group had a higher left ventricular mass index (140.1 \pm 10.7 vs $\left.123.5 \pm 11.9 \mathrm{~g} / \mathrm{m}^{2}, p<0.05\right)$ and VOI $(43.3 \pm 8.3$ vs $40.1 \pm 8.9 \mathrm{mmHg}, p<0.05)$. VOI was the significant correlated factor to left ventricular mass index in both middle-aged $(r=0.70, p<0.01)$ and elderly $(r=0.78, p<0.01)$ groups. ${ }^{28}$ Another observational cross-sectional study compared the correlation of target organ damage and VOI between hypertensive patients with and without metabolic syndrome. ${ }^{15}$ The parameters to evaluate target organ damage were defined as albumin-to-creatinine ratio, left ventricular mass index and carotid intima-media thickness. ${ }^{15}$ Compared with the non-metabolic dysfunction group, this study demonstrated a significant association between VOI and target organ damage in the metabolic syndrome group, ${ }^{15}$ while to some extent the significant relationship of VOI and adverse outcome was clarified indefinitely with the 
Table 3 Association Between VOI and Ischemic Stroke in Subgroups

\begin{tabular}{|c|c|c|c|c|c|}
\hline & Number & $\mathbf{H R}^{\mathbf{a}}$ & $95 \% \mathrm{Cl}^{\mathrm{a}}$ & $p$-value & $p$ for Interaction \\
\hline Sex & & & & & 0.47 \\
\hline Male & 1475 & 1.21 & $(1.08-1.33)$ & $<0.001$ & \\
\hline Female & 1840 & 1.11 & $(1.07-1.22)$ & 0.003 & \\
\hline Smoking status & & & & & 0.15 \\
\hline No & 2382 & 1.11 & $(1.04-1.22)$ & 0.015 & \\
\hline Yes & 933 & 1.20 & $(1.03-1.33)$ & $<0.001$ & \\
\hline Alcohol consumption & & & & & 0.67 \\
\hline No & 2977 & 1.14 & $(1.04-1.22)$ & 0.004 & \\
\hline Yes & 338 & 1.17 & $(1.09-1.24)$ & $<0.001$ & \\
\hline Diabetes mellitus & & & & & 0.38 \\
\hline No & 2810 & I.II & $(I .0 I-1.22)$ & $<0.001$ & \\
\hline Yes & 505 & 1.21 & $(1.09-1.33)$ & $<0.001$ & \\
\hline SBP & & & & & 0.32 \\
\hline$<140 \mathrm{mmHg}$ & 2361 & 1.19 & $(1.07-1.32)$ & 0.003 & \\
\hline$\geq 140 \mathrm{mmHg}$ & 954 & 1.11 & $(1.09-1.22)$ & 0.005 & \\
\hline Body mass index & & & & & 0.57 \\
\hline$<25 \mathrm{~kg} / \mathrm{m}^{2}$ & 2127 & 1.11 & $(1.03-1.21)$ & 0.010 & \\
\hline$\geq 25 \mathrm{~kg} / \mathrm{m}^{2}$ & 1188 & 1.22 & $(1.09-1.33)$ & $<0.001$ & \\
\hline ACEI & & & & & 0.43 \\
\hline No & 3103 & 1.09 & $(1.01-1.22)$ & $<0.001$ & \\
\hline Yes & 212 & 1.19 & $(1.07-1.32)$ & $<0.001$ & \\
\hline ARB & & & & & 0.39 \\
\hline No & 2669 & I.II & $(1.05-1.22)$ & 0.005 & \\
\hline Yes & 646 & 1.20 & $(1.09-1.32)$ & 0.004 & \\
\hline $\mathrm{CCB}$ & & & & & 0.37 \\
\hline No & 2659 & 1.11 & $(1.04-1.22)$ & 0.004 & \\
\hline Yes & 656 & 1.20 & $(1.09-1.32)$ & $<0.001$ & \\
\hline
\end{tabular}

Notes: ${ }^{\mathrm{a}} \mathrm{HR}$ and $95 \% \mathrm{Cl}$ were derived from multivariate Cox regression models. Multivariate Cox regression models accounted for age, sex, body mass index, smoking status, alcohol consumption, systolic blood pressure, total cholesterol, triglyceride, low density lipid cholesterol, high density lipid cholesterol, glucose, estimated glomerular filtration rate, diabetes mellitus, coronary artery disease, antihypertensive medication including angiotensin converting enzyme inhibitor, angiotensin receptor blocker and calcium channel blocker.

Abbreviations: SBP, systolic blood pressure; VOI, vascular overload index; LDL-C, low density lipid cholesterol; HDL-C, high density lipid cholesterol; ACEl, angiotensin converting enzyme inhibitor; $\mathrm{ARB}$, angiotensin receptor blocker; $\mathrm{CCB}$, calcium channel blocker, $\mathrm{HR}$, hazard ratio; $95 \% \mathrm{Cl}, 95 \% \mathrm{confidence}$ interval.

confounders of higher level of cholesterol and metabolic system dysfunction. As reviewed and studied elsewhere, aging, ${ }^{29}$ blood pressure control, ${ }^{3,7}$ cholesterol levels ${ }^{30}$ and lifestyle ${ }^{28,31}$ play non-negligible roles in the process of adverse cardiovascular and cerebrovascular outcomes, in particular the incidence of ischemic stroke. ${ }^{23,24}$ Our study moved this field forward by administrating a longitudinal design and excluding interaction effects after adjusting confounders, proving the precise and rational causal relationship between increased VOI and ischemic stroke.

\section{Strength and Limitations}

Strong points of this study are the comparatively large sample size in a longitudinal study and the annual follow-ups to record the endpoint. Furthermore, we ran the stratification analysis based on covariables to confirm the association between VOI and new-onset ischemic stroke in different subgroups and confirmed the main analysis results. However, our current finding must also be interpreted within the context of their limitations. First, we included a high prevalence of $15.2 \%$ of diabetic participants in analysis, whose pathologic vessel change and 
vulnerable vessel status may have a pathophysiologic effect on the value of VOI. However, the stratification analysis (Table 3) provided a reliable instruction on the unbiased result between diabetic and non-diabetic participants. Second, residual confounding effect might exist due to the multiple mechanism of hypertension. Third, this study was only administered in a single region and Chinese ethnicity, which limits extrapolation to other populations. Fourth, as VOI is a parameter reflecting arterial stiffness, we did not perform the measurements of pulse wave velocity and augmentation index as the validation approach, which might be a limitation of this study. Fifth, high sensitive C-reactive protein, the biomarker of atherosclerosis, is associated with arterial stiffness and cardiovascular risks, which were not measured in our current study. ${ }^{32,33}$ Further studies are expected to make deeper clarification on the association between VOI and ischemic stroke.

\section{Conclusions}

This longitudinal study demonstrated the significant association between VOI and new-onset ischemic stroke. VOI is associated with new-onset ischemic stroke in an elderly population with hypertension. Elevated VOI is a correlated risk factor and increases the probability of new-onset ischemic stroke. As a non-invasive and feasible parameter, VOI can be a predictive factor of increasing risk of newonset ischemic stroke for clinical practice.

\section{Acknowledgments}

We would like to appreciate all the participants and medical staff in the observation center who contributed to this study.

\section{Funding}

This study was supported by Guangdong Provincial People's Hospital Clinical Research Fund (Y012018085), the Key Area R\&D Program of Guangdong Province (No.2019B020227005), Science and Technology Plan Program of Guangzhou (No. 201803040012), the Fundamental and Applied Basic Research Foundation Project of Guangdong Province (2020A1515010738), and the Climbing Plan of Guangdong Provincial People's Hospital (DFJH2020022).

\section{Disclosure}

The authors report no relationships that could be construed as a conflict of interest.

\section{References}

1. Baski AJ, Treibel TA, Davies JE, et al. A meta-analysis of the mechanism of blood pressure change with aging. $\mathrm{J} \mathrm{Am}$ Coll Cardiol. 2009;54:2087-2092. doi:10.1016/j.jacc.2009.06.049

2. Unger T, Borghi C, Charchar F, et al. 2020 International society of hypertension global hypertension practice guidelines. J Hypertens. 2020;38:982-1004. doi:10.1097/HJH.0000000000002453

3. Williams B, Mancia G, Spiering W, et al. 2018 ESC/ESH guidelines for the management of arterial hypertension: the Task Force for the management of arterial hypertension of the European Society of Cardiology and the European Society of Hypertension. J Hypertens. 2018;36:1953-2041.

4. Chirinos JA, Segers P, Hughes T, Townsend R. Large-artery stiffness in health and disease: JACC state-of-the-art review. $J$ Am Coll Cardiol. 2019;74:1237-1263. doi:10.1016/j.jacc.2019.07.012

5. Liu LS. [2010 Chinese guidelines for the management of hypertension]. Zhonghua Xin Xue Guan Bing Za Zhi. 2011. Chinese.

6. Whelton PK, Carey RM, Aronow WS, et al. 2017 ACC/AHA/AAPA/ $\mathrm{ABC} / \mathrm{ACPM} / \mathrm{AGS} / \mathrm{APhA} / \mathrm{ASH} / \mathrm{ASPC} / \mathrm{NMA} / \mathrm{PCNA}$ guideline for the prevention, detection, evaluation, and management of high blood pressure in adults: a report of the American College of Cardiology/ American Heart Association Task Force on Clinical Practice Guidelines. J Am Coll Cardiol. 2018;71:e127-e248. doi:10.1016/j. jacc.2017.11.006

7. Yu YL, Liu L, Huang JY, et al. Association between systolic blood pressure and first ischemic stroke in the Chinese older hypertensive population. $J$ Int Med Res. 2020;48:1-12. doi:10.1177/ 0300060520920091

8. Meschia JF, Bushnell C, Boden-Albala B, et al. Guidelines for the primary prevention of stroke: a statement for healthcare professionals from the American Heart Association/American Stroke Association. Stroke. 2014;45:3754-3832. doi:10.1161/STR.00000000000000046

9. Kannel WB, Schwartz MJ, McNamara PM. Blood pressure and risk of coronary heart disease: the Framingham study. Dis Chest. 1969;56:43-52. doi:10.1378/chest.56.1.43

10. Kannel WB, Wolf PA, Verter J, McNamara PM. Epidemiology assessment of the role of blood pressure in stroke. JAMA. 1996;276:1269-1278. doi:10.1001/jama.1996.03540150071040

11. Kannel WB, Gordon T, Schwartz MJ. Systolic versus diastolic blood pressure and risk of coronary heart disease: the Framingham study. Am J Cardiol. 1971;27:335-346. doi:10.1016/0002-9149(71)90428-0

12. de Simone G, Pasanisi F. Pressione arteriosa sistolica, diastolica e differenziale: aspetti fisiopatologici [Systolic, diastolic and pulse pressure: pathophysiology]. Ital Heart J Suppl. 2001;2:359-362.

13. Frankin SS, Weber MA. Measuring hypertensive cardiovascular risk: the vascular overload concept. Am Heart J. 1994;128:793-803. doi:10.1016/0002-8703(94)90278-X

14. Cai XQ, Zhang LY, Huang XD, Shen YH, Xie LD. The relationship between vascular overload index and intima media thichness of carotid artery in hypertensive patients. Chin $J$ Hypertens. 2014;22:830-835.

15. Wang YR, Huang XY, Wei JR. Relationship between blood vessel overload index and target-organ damage in patients with essential hypertension combining with metabolic dyndrome. South Chin J Cardiol. 2009;15:446-449.

16. Ketteler M, Block GA, Evenepoel P, et al. Executive summary of the 2017 KDIGO Chronic Kidney Disease-Mineral and Bone Disorder (CKD-MBD) guideline update: what's changed and why it matters. Kidney Int. 2017;92:26-36. doi:10.1016/j.kint.2017.04.006

17. Baluch UT, Kiani I, Zaib-un-nisa BM. Association of dyslipidemia and ischemic stroke. Ann Pak Inst Med Sci. 2008;4:165-167.

18. Zheng L, Sun Z, Zhang X, Li J, Hu D, Sun Y. The association between glomerular filtration rate and stroke in hypertensive patients in rural areas of China. J Hypertens. 2012;30:901-907. doi:10.1097/ HJH.0b013e328352abc0 
19. Zhang Y, Li J, Liu C, et al. High-density lipoprotein cholesterol and the risk of first ischemic stroke in a Chinese hypertensive population. Clin Interv Aging. 2021;2021:801-810. doi:10.2147/CIA.S295252

20. Groenwold RHH, Goeman JJ, Cessie SL, Dekkers OM. Multiple testing: when is many too much? Eur J Endocrinol. 2021;184:E11E14. doi:10.1530/EJE-20-1375

21. SHEP Cooperative Research Group. Prevention of stroke by antihypertensive drug treatment in older persons with isolated systolic hypertension. Final results of the Systolic Hypertension in the Elderly Program (SHEP). JAMA. 1991;3255-3264.

22. Perry HM, Davis BR, Price TR, et al. Effect of treating isolated systolic hypertension on the risk of developing various types and subtypes of stroke. The Systolic Hypertension in the Elderly Program (SHEP). JAMA. 2000;284:465-471. doi:10.1001/jama.284.4.465

23. Group SR, Wright JT, Williamson JD, et al. A randomized trial of intensive versus standard blood-pressure control. Ann Int Conf. 2015;2015:2103-2116. doi:10.1109/EMBC.2015.7318803

24. Williamson JD, Supiano MA, Applegate WB, et al. Intensive vs standard blood pressure control and cardiovascular disease outcomes in adults aged $>/=75$ years: a randomized clinical trial. JAMA. 2016;2016:2673-2682.

25. Cernes R, Zimlichman R, Shargorodsky M. Arterial elasticity in cardiovascular disease: focus on hypertension, metabolic syndrome and diabetes. Adv Cardiol. 2008;45:65-81.
26. Intengan HD, Deng LY, Li JS, Schiffrin EL. Mechanics and composition of human subcutaneous resistance arteries in essential hypertension. Hpertension. 1999;33:569-574. doi:10.1161/01.HYP.33.1.569

27. Beevers G, Lip GYP, O'Brien E. The pathophysiology of hypertension. BMJ. 2001;322:912-916. doi:10.1136/ bmj.322.7291.912

28. Reynolds K, Lewis LB, Nolen JD, Kinney GL, Sathya B, He J. Alcohol consumption and risk of stroke: a meta-analysis. JAMA. 2003;289:579-588. doi:10.1001/jama.289.5.579

29. Cai W, Zhang K, Li P, et al. Dysfunction of the neurovascular unit in ischemic stroke and neurodegenerative diseases: and aging effect. Aging Res Rev. 2017;34:77-87. doi:10.1016/j.arr.2016.09.006

30. Ansell BJ. Cholesterol, stroke risk, and stroke prevention. Curr Atheroscler Rep. 2000;2:92-96. doi:10.1007/s11883-000-0101-5

31. Hankey GJ. Smoking and risk of stroke. J Cardiovasc Risk. 1999;6:207-211. doi:10.1177/204748739900600403

32. Tibaut M, Caprnda M, Kubatka P, et al. Markers of atherosclerosis: part 1 - serological markers. Heart Lung Circ. 2018;28:667-677. doi:10.1016/j.hlc.2018.06.1057

33. Mozos I, Jianu D, Gug C, Stoian D. Links between high-sensitivity C-reactive protein and pulse wave analysis in middle-aged patients with hypertension and high normal blood pressure. Dis Markers. 2019;2019:1-9. doi:10.1155/2019/2568069
Clinical Interventions in Aging

\section{Publish your work in this journal}

Clinical Interventions in Aging is an international, peer-reviewed journal focusing on evidence-based reports on the value or lack thereof of treatments intended to prevent or delay the onset of maladaptive correlates of aging in human beings. This journal is indexed on PubMed Central, MedLine, CAS, Scopus and the Elsevie
Dovepress

Bibliographic databases. The manuscript management system is completely online and includes a very quick and fair peer-review system, which is all easy to use. Visit http://www.dovepress.com/ testimonials.php to read real quotes from published authors. 